R. SHIBATA

KODAI MATH. J.

$2(1979), 38-42$

\title{
A NOTE ON ASYMPTOTIC UNBIASEDNESS OF ESTIMATES
}

\author{
By Ritei Shibata
}

\section{$\S 1$. Introduction.}

In estimation theory the concept of asymptotic unbiasedness of estimates is an important extension of ordinary unbiasedness. An estimator is intuitively called asymptotic ally unbiased when the bias function converges to zero with the smaller order than that of the variance for each parameter. This definition, however, admits so called super-efficient estimator whose asymptotic variance never exceeds the Cramér-Rao lower bound and is below it at some parameter ([10], [13]). Walker [12] showed that if the derivative of the bias function also converges to zero, such estimator is excluded. Another approach by Rao [7], [8] and Schmetterer [9] is that if an estimator is asymptotic normal and its convergence is uniform in the compact neighbourhood of each parameter, it is not super-efficient.

In this paper combining these two approaches we will propose a new definition of asymptotic unbiasedness and show that the Cramér-Rao inequality asymptotically holds in a general framework. Although the proposed class of estimates is wider, it is easier to verify the conditions because the definition depends only on how the bias function converges to zero and no asymptotic distribution or derivative of the bias function is needed.

\section{$\S 2$. Asymptotic unbiasedness.}

Let $X_{n}=\left(x_{1}, x_{2}, \cdots, x_{n}\right)$ be a sequence of $n$ random variables distributed according to the joint probability density function $f\left(X_{n}, \theta\right)$ with respect to a $\sigma$ finite measure $\mu\left(d X_{n}\right)$ on $R^{n}$, where $\theta$ is a parameter which can take any value in an open interval $\Theta$. We assume the following regularity condition on the density $f\left(X_{n}, \theta\right)$.

Condition 1. For each $\theta \in \Theta$ there exists a positive sequence $c_{n}$ (which may depend on $\theta$ ) such that

$$
\tilde{I}(\theta)=\varlimsup_{h \rightarrow+0} \frac{1}{h^{2}} \varlimsup_{n \rightarrow \infty} E_{\theta}\left(\frac{f\left(X_{n}, \theta+h / c_{n}\right)-f\left(X_{n}, \theta\right)}{f\left(X_{n}, \theta\right)}\right)^{2}
$$

Received August 17, 1977. 
is posituve finte, where the expectation $E_{\theta}$ is taken on the support of $\int\left(X_{n}, \theta\right)$.

As is well known, with some regurality conditions $I(\theta)$ is represented as

$$
\bar{I}(\theta)=\varlimsup_{h \rightarrow+0} \frac{1}{h^{2}} \varlimsup_{n \rightarrow \infty} I_{n}\left(\theta, \theta+h / c_{n}\right)
$$

by the Kullback Leibler information

$$
I_{n}\left(\theta_{1}, \theta_{2}\right)=E_{\theta_{1}}\left\{\log \frac{f\left(X_{n}, \theta_{1}\right)}{f\left(X_{n}, \theta_{2}\right)}\right\} \quad \theta_{1}, \theta_{2} \in \Theta
$$

(see [4]).

Condition 1 is satisfied if $x_{1}, x_{2}, \cdots, x_{n}$ are independent, identically distr1buted and the Fisher information

$$
I(\theta)=E_{\theta}\left(\frac{\partial}{\partial \theta} \log f\left(X_{1}, \theta\right)\right)^{2}
$$

is positive finite, in fact putting $c_{n}=\sqrt{n}$ we have

$$
\begin{aligned}
\bar{I}(\theta) & =\varlimsup_{h \rightarrow+0} \frac{1}{h^{2}} \varlimsup_{n \rightarrow \infty}\left\{\left(E_{n}\left(\frac{f\left(X_{1}, \theta+h / \sqrt{n}\right)-f\left(X_{1}, \theta\right)}{f\left(X_{1}, \theta\right)}\right)^{2}+1\right)^{n}-1\right\} \\
& =\lim _{h \rightarrow 0} \frac{1}{h^{2}}\left[\lim _{n \rightarrow \infty}\left\{I(\theta) \frac{h^{2}}{n}+1\right\}^{n}-1\right] \\
& =\lim _{h \rightarrow 0} \frac{1}{h^{2}}-\left[e^{I(\theta) h^{2}}-1\right]=I(\theta) .
\end{aligned}
$$

In this paper we will consider estimation of the parameter $\theta$. Condition 1 implies that $\left|c_{n}\left(T_{n}-\theta\right)\right|$ is bounded away from zero in probability for any estimator $T_{n}\left(X_{n}\right)$ of $\theta([11])$. That is, the possible highest order of consistency is $O\left(c_{n}\right)$. Assuming the existence of the bias function $b_{n}(\theta)=E_{\theta}\left(T_{n}-\theta\right)$ we will define asymptotic unbiasedness of $T_{n}$ in such a general framework.

Definition. An estimator $T_{n}$ of $\theta$ is said to be asymptotically unbiased (from the right) at $\theta$ if

$$
c_{n} b_{n}(\theta) \longrightarrow 0 \text { as } n \longrightarrow \infty,
$$

and one of the following conditions is satisfied

(1) for any small $h>0$

$$
c_{n} b_{n}\left(\theta+h / c_{n}\right) \longrightarrow 0 \text { as } n \longrightarrow \infty,
$$

(2) there exists a positive sequence $\delta_{n}$ which converges to zero with $o\left(1 / c_{n}\right)$ and

$$
\left(b_{n}\left(\theta+\delta_{n}\right)-b_{n}(\theta)\right) / \delta_{n} \longrightarrow 0 \text { as } n \longrightarrow \infty .
$$

The asymptotic unbiasedness from the left is similarly defined and the dis- 
cussion is the same. The condition (2.2) requires that the bias of $T_{n}$ vanishes as $n \rightarrow \infty$ with the smaller order than that of variance and the condition (2.3) or (2.4) requires some uniformity of the convergence.

Proposition 1. If one of the following conditions is satisfied, then $T_{n}$ is asymptotic ally unbrased,

a) both $c_{n} b_{n}(\theta)$ and the dervative $b_{n}^{\prime}(\theta)$ converge to zero as $n \rightarrow \infty$ (Walker [12]),

b) the distribution function $F_{n}(t, \theta)$ of $c_{n}\left(T_{n}-\theta\right)$ converges to a normal distribution function unformly in the compact neighbourhood of $\theta$ for each fixed $t$ and the $(1+\delta)$ th absolute moment of $F_{n}(t, \theta)$ is bounded for some $\delta>0$ (Rao and Schmetterer [7], [8], [9]),

c) $c_{n} b_{n}(\theta)$ converges to zero as $n \rightarrow \infty$ unformly in the compact neighbourhood of $\theta$ (Ibragımov and Khas'minskii [5]),

d) $c_{n} b_{n}(\theta)$ converges to zero as $n \rightarrow \infty$ and is equicontmmous.

Proof. If a) holds, we can choose a positive sequence $\hat{\delta}_{n}=O\left(1 / c_{n}\right)$ such that for any $\varepsilon>0$ there exists a positive integer $N$ and

$$
\left|\frac{b_{n}\left(\theta+\delta_{n}\right)-b_{n}(\theta)}{\delta_{n}}-b_{n}^{\prime}(\theta)\right|<\varepsilon, \quad n \geqq N,
$$

so that (2.4) follows. The condition b) implies c), and (2.2) and (2.3) follow from c) or d).

Remark. The moment condition in b) is not so strong since our main concern is in the mean squared error and it is sufficient to consider only estimates whose second moments are bounded.

The above proposition shows that our definition is very wide. Next we will show that the Cramér-Rao inequality holds for such estimates.

Theorem. Assume Condition 1. If $T_{n}$ is asymptotically unbrased then the following inequality holds,

$$
\lim _{n \rightarrow \infty} E_{\theta}\left(c_{n}\left(T_{n}-\theta\right)\right)^{2} \geqq 1 / \bar{I}(\theta)
$$

Proof. From the Chapman-Robbins inequality [3], we have

$$
E_{\theta}\left(c_{n}\left(T_{n}-\theta\right)\right)^{2} \geqq \frac{c_{n}^{2}\left(b_{n}(\theta+\alpha)-b_{n}(\theta)+\alpha\right)^{2}}{E_{\theta}\left(\frac{f\left(X_{n}, \theta+\alpha\right)-f\left(X_{n}, \theta\right)}{f\left(X_{n}, \theta\right)}\right)^{2}}
$$

for any $\theta, \theta+\alpha \in \Theta$, where $\alpha>0$. If $T_{n}$ satisfies (2.2) and (2.3), putting $\alpha=h / c_{n}$ and dividing the denominator and the numerator of the right side of (2.5) by $h^{2}$, we have the desired result from Condition 1 . We can obtain the result putting $\alpha=\hat{o}_{n}$ in the same manner if $T_{n}$ satisfies (2.2) and (2.4).

Example. Let $x_{2}$ have the mean 


$$
\mu_{\iota}(\theta)=E_{\theta} x_{\imath}= \begin{cases}\theta-(1-\theta) \theta^{\imath-1} & \text { if }|\theta| \leqq 1 \\ \theta-(1-\theta) \theta^{-\imath} & \text { otherwise },\end{cases}
$$

where $-\infty<\theta<\infty$. Consider an estimator $T_{n}=\frac{1}{n} \sum_{i=1}^{n} x_{\imath}$ of $\theta$, then the bras function of $T_{n}$ becomes

$$
b_{n}(\theta)= \begin{cases}\frac{1}{n}\left(\theta^{n}-1\right) & \text { if } \quad|\theta| \leqq 1 \\ \frac{1}{n}\left(1-\theta^{-n}\right) & \text { otherwise }\end{cases}
$$

and the derivative is

$$
b_{n}^{\prime}(\theta)= \begin{cases}\theta^{n-1} & \text { if }|\theta| \leqq 1 \\ \theta^{-n-1} & \text { otherwise }\end{cases}
$$

Since $b_{n}^{\prime}(1)=1$ and $b_{n}^{\prime}(-1)=(-1)^{n-1}$, the condition a) in Proposition 1 is not satisfied at $\theta=1$ or -1 . Therefore we can not apply Walker's result. Furthermore if $x_{\imath}$ satisfies the following linear equation,

$$
x_{i}=x_{1}+\mu_{i}(\theta)-\mu_{1}(\theta)
$$

then

$$
T_{n}-\theta=x_{1}+b_{n}(\theta)-\mu_{1}(\theta) .
$$

Unless $x_{1}$ is normal, $T_{n}$ is not asymptotically unbiased in the sense of Rao and Schmetterer since $T_{n}$ is not asymptotically normal.

However (2.2) and (2.4) hold for any positive sequence $\delta_{n}$ such as $n \hat{\delta}_{n} \rightarrow \infty$ and $\delta_{n} c_{n} \rightarrow 0$ when the Condition 1 is satisfied and $c_{n}=o(n)$, and $T_{n}$ is asymptotically unbiased in our sense.

\section{REFERENCES}

[1] Bahadir, R.R., On the asymptotic efficiency of tests and estimators, Sankhya, 22 (1960), 229-252.

[2] Bahadur, R.R., On Fisher's bound for asymptotic variances, Ann. Math. Statist., 35 (1964), 1545-1552.

[3] Chapman, D. G. AND H. Robbins, Minimum variance estimation without regularity assumptions, Ann. Math. Stat1st, 22 (1951), 581-586.

[4] Daviels, H.E., The asymptotic efficiency of a maximum likelihood estimator, Proc. Fourth Berkley Symp. Math. Statıst. Prob., I (1961), 151-164.

[5] IbRagimov, I. A. A.ND R.Z. Khas'MivskiI, Information-theoretic inequalities and superefficient estimates, Problems of Inform. Transmission, 9 (1973), 53-67.

[6] RAO, C.R., On Fisher's bound to asymptotic variance of a consistent estimate, Sankhya, 15 (1955), 331-342.

[7] RaO, C.R., Asymptotic efficiency and limitıng information, Proc. Fourth Berkley 
Symp. Math. Statist. Prob., 1 (1961), 531-536.

8. RAo, C.R., Criteria of estimation in large samples, Sankhya, Series A, 25 (1963), 189-206.

19. Scinetrtrer, L., On the asymptotic efficiency of estimates, Research Papers in Statistics (Festschrift J. Neyman), John Wiley, London (1966), 301-317.

[10 SchanetTerer, L., Introduction to Mathematical Statistics, Springer, Berlin (1974), 295-353.

[11] Thkeucil, K., Asymptotic theory of statıstical estimation, Kyōiku Shuppan, Tokyo, (1974) (in Japanese).

[12: Walier, A. M., A note on the asymptotic efficiency of an asymptotically normal estımator sequence, J. Roy. Stat. Soc. B, 25 (1963), 195-200.

[13: Zacks, S., The Theory of Statistical Inference, John Wiley, New York, (1971).

Department of Mathematics

TOKYO Institute of TECHNOLOGY

Oh-okayama, Meguro-ize, Tokyo

JAPAN 